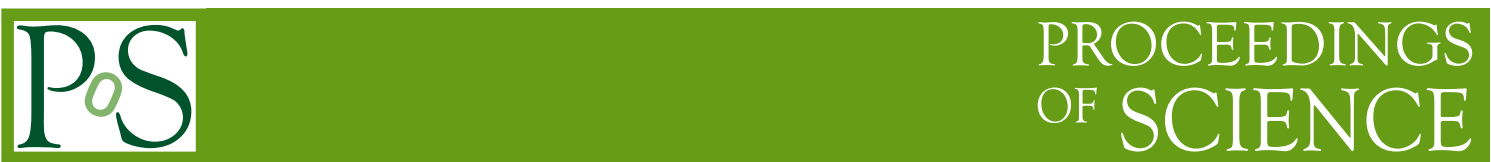

\title{
Large-scale, intergalactic magnetic and radiation fields
}

\section{Andrii Neronov*}

ISDC Data Centre for Astrophysics, Ch. d'Ecogia 16, 1290 Versoix, Switzerland

E-mail: Andrii.Neronov@unige.ch

I review the recent evidence for existence of large scale intergalactic magnetic fields (IGMF) derived from the gamma-ray observations of distant $\mathrm{TeV}$ gamma-ray loud blazars. Non-observation of the signal from electromagnetic cascade initiated by $\mathrm{TeV}$ gamma-rays in the intergalactic medium imposes a lower bound on IGMF strength at the level of $B>10^{-17} \mathrm{G}$ if the IGMF correlation length is larger than $\sim 1 \mathrm{Mpc}$ and the cascade signal is assumed to be suppressed due to the time delay of the cascade emission. The bound is $B>10^{-16} \mathrm{G}$ for Mpc-scale correlation lenght if the cascade emission is not observed due to the extended nature of the cascade emission. The bound improves as $\lambda^{-1 / 2}$ if magnetic field correlation length is much smaller than a Mega-parsec. This lower bound constrains models for the origin of cosmic magnetic fields.

25th Texas Symposium on Relativistic Astrophysics - TEXAS 2010

December 06-10, 2010

Heidelberg, Germany

\footnotetext{
*Speaker.
} 


\section{Introduction}

The presence of magnetic fields in galaxies and galaxy clusters plays a key role in present day astrophysical studies. However, the origin of these fields remains largely uncertain $[1,2$, 3,4]. A commonly accepted hypothesis is that relatively strong galactic and cluster magnetic fields result from the amplification of much weaker pre-existing "seed" fields via compression and turbulence/dynamo amplification in the course of structure formation process [5].

The origin of these seed magnetic fields is unknown. It is possible that the seed fields are produced locally in (proto)galaxies via the so-called "Biermann battery" mechanism [6,7]. Otherwise, the seed fields might be of primordial origin, i.e. produced at the moments of phase transitions in the Early Universe [2,3]. Constraints on the nature of the seed fields could potentially be derived from the measurements of weak magnetic fields in the intergalactic medium which are not amplified by the action of different types of dynamos.

Most of the attempts to measure IGMF have concentrated up to recently on the measurement of Faraday rotation of polarized radio emission from distant sources and/or search for the magnetic field-related features in the CMB power spectrum and polarization[1, 2, 3, 4]. A new handle on the EGMF measure, using the cascade emission from blazars, is now emerging as an alternative probe. In this method, multi-TeV $\gamma$-rays from distant $(>100 \mathrm{Mpc}$ ) blazars attenuate through pair production interactions on the extragalactic background light (EBL), leading to the development of electromagnetic cascades $[8,9,10,11,12,13,14,15,16]$. Angular pattern of the secondary cascade emission from $e^{+} e^{-}$pairs deposited in the intergalactic medium in result of the pair production depends on the EGMF strength. Detection (non-detection) of the cascade emission signal from known $\mathrm{TeV} \gamma$-ray emitting blazars could result in the measurement of (lower bound on) the strength of magnetic field in the intergalactic space along the line of sight toward these blazars. The first application of this method for deriving lower bounds on the EGMF have been carried out [17, 18, 19], suggesting that a measure of the EGMF may finally soon be within reach.

\section{TeV gamma-ray induced cascades in intergalactic medium}

$\gamma$-rays with energies above $\sim 1 \mathrm{TeV}$ can not propagate over cosmological distances because of absorption due to interactions with diffuse Extragalactic Background Light (EBL) [20, 21, 22, 23]. The mean free path of $\gamma$-rays of energy $E_{\gamma_{0}}$ through EBL is $D_{\gamma} \simeq 80 \kappa\left[E_{\gamma_{0}} / 10 \mathrm{TeV}\right]^{-1} \mathrm{Mpc}$, where $\kappa \sim 1$ is a numerical factor which accounts for uncertainties of the measurements and modeling of the EBL [14]. Interactions of multi-TeV $\gamma$-rays with the EBL lead to the deposition of electronpositron pairs in the intergalactic space. These $e^{+} e^{-}$pairs emit secondary cascade $\gamma$-rays via Inverse Compton (IC) scattering of CMB photons. Typical energies for the IC photons emitted by electrons of energy $E_{e} \simeq E_{\gamma_{0}} / 2$ are $E_{\gamma}=(4 / 3) \varepsilon_{C M B}\left(E_{e} / m_{e} c^{2}\right)^{2} \simeq 88\left[E_{\gamma_{0}} / 10 \mathrm{TeV}\right]^{2} \mathrm{GeV}$ where $\varepsilon_{C M B}=6 \times 10^{-4} \mathrm{eV}$ is the typical energy of $\mathrm{CMB}$ photons and $E_{e}, m_{e}$ are the energy and mass of electron. Pairs loose energy on IC scattering on the distance scale $D_{e} \simeq 10^{23}\left(E_{e} / 10 \mathrm{TeV}\right)^{-1} \mathrm{~cm}$, which is much smaller than the $\gamma$-ray mean free path $D_{\gamma}$. Power removed from the primary $\gamma$-ray beam is transferred to the cascade $\gamma$-ray emission.

If magnetic fields, which deviate electron and positron trajectories, are negligibly small, the IC emission from the electromagnetic cascade contributes to the primary point $\gamma$-ray source flux. 
Otherwise, if magnetic fields along the path of development of the cascade are strong enough to deviate the trajectories of the pairs, the cascade emission appears as extended and delayed emission around the initial point source.

The deflection angle $\delta$ depends on the correlation length of the magnetic field. If $\lambda_{B} \gg D_{e}$, the motion of electrons/positrons can be approximated by the motion in a homogeneous magnetic field. In this case $\delta \simeq D_{e} / R_{L} \simeq 3 \times 10^{-4}\left[B / 10^{-16} \mathrm{G}\right]\left[E_{e} / 10 \mathrm{TeV}\right]^{-2}$ is a ratio of $D_{e}$ to the Larmor radius $R_{L}$. If $\lambda_{B} \ll D_{e}$, electron deflections are describable by diffusion in angle, so that the deflection angle is $\delta=\sqrt{D_{e} \lambda_{B}} / R_{L} \simeq 5 \times 10^{-5}\left[E_{e} / 10 \mathrm{TeV}\right]^{-3 / 2}\left[B / 10^{-16} \mathrm{G}\right]\left[\lambda_{B} / 1 \mathrm{kpc}\right]^{1 / 2}$. The size of the extended cascade source is estimated as $\Theta_{\text {ext }} \simeq \delta / \tau$, where $\tau=D / D_{\gamma}$ is the optical depth for $\gamma$-rays from a source at a distance $D$ with respect to absorption on EBL [14]. The time delay of the cascade signal is given by $T_{\text {delay }} \simeq(1+z) D_{\gamma} \delta^{2}\left(1-D_{\gamma} / D\right) / 2 \simeq 7 \times 10^{5}\left(1-\tau^{-1}\right)(1+$ $z)^{-5}\left[E_{\gamma} / 0.1 \mathrm{TeV}\right]^{-5 / 2}\left[B / 10^{-18} \mathrm{G}\right]^{2} \mathrm{~s}$ in the case of magnetic fields with large correlation length and $T_{\text {delay }} \simeq 10^{4}\left(1-\tau^{-1}\right)(1+z)^{-2}\left[E_{\gamma} / 0.1 \mathrm{TeV}\right]^{-2}\left[B / 10^{-18} \mathrm{G}\right]^{2}\left[\lambda_{B} / 1 \mathrm{kpc}\right] \mathrm{s}$ in the case of magnetic fields with short correlation legngth $\lambda_{B} \ll D_{e}$.

Recent attempts of detection of the cascade emission contribution in the GeV-band spectra of blazars have not resulted in positive detecitons up to now [17, 18, 19, 24]. The upper limits dervied from the Fermi/LAT data are below the expected cascade flux calculated under the assumption of zero IGMF. The $\mathrm{GeV}$ band cascade emission produced in result of interactions of $\mathrm{TeV} \gamma$-rays with EBL could be suppressed in two ways. First, the cascade emission might be not detectable if the signal is stretched in time by the time delay $T_{\text {delay }}$ which is much larger than the typical period of source activity. Otherwise, the cascade emission might be not detectable if the cascade signal is much more extended than the point spread function of a $\gamma$-raytelescope.

The cascade signal is expected to be strongest for the $\mathrm{TeV}$ blazars with hard intrinsic spectra situated at large (for VHE $\gamma$-ray astronomy) redshifts. These are the same blazars which provide best constraints on the EBL, e.g. 1ES 0229+200, 1ES 0347-121, 1ES 1101-232. The cascade emission could be detectable only if the primary soruce spectrum extends to the multi- TeV band. The time delay of the cascade signal is uncertain by many orders of magneitude. If IGMF are relatively strong, at the level of $10^{-15} \mathrm{G}$ and above, the time delay at $0.11-10 \mathrm{GeV}$ energies could reach the scales comparable to the life time of AGN. Otherwise, if EGMF is $10^{-18} \mathrm{G}$ and weaker, the time delay in the same energy band could be in the range of months-to-years. This means that the cascade $\mathrm{GeV}$ emission could appear either quasi-simultaneously with the direct $\mathrm{TeV}$ emission or be present as a persistent emission uncorrelated with the $\mathrm{TeV}$ band direct emission. The uncertainty of the time delay implies that quasi-simultaneous observations of blazars in the $\mathrm{GeV}$ and $\mathrm{TeV}$ band should be preferentially used to derive constraints on EGMF. Reports on such observations have started to appear over the last year in the literature.

The studies reported in references [19] and [24] have used the simultaneous observations of $\mathrm{TeV}$ blazars by Fermi and by the ground-based $\gamma$-ray telescopes to derive the lower bounds on IGMF strength imposed by the non-observation of the cascade emission in the $\mathrm{GeV}$ band, assuming that the cascade emission is suppressed due to the time delay of the cascade signal (see right panel of Fig. 1). The lower bound on IGMF derived under this assumption is at the level of $10^{-17} \mathrm{G}$ if the magnetic field correlation length is larger than $\sim 1 \mathrm{Mpc}$. If the correlation length of IGMF is smaller, the bound improves at $\lambda^{-1 / 2}$, because stronger magnetic fields are needed to deflect 

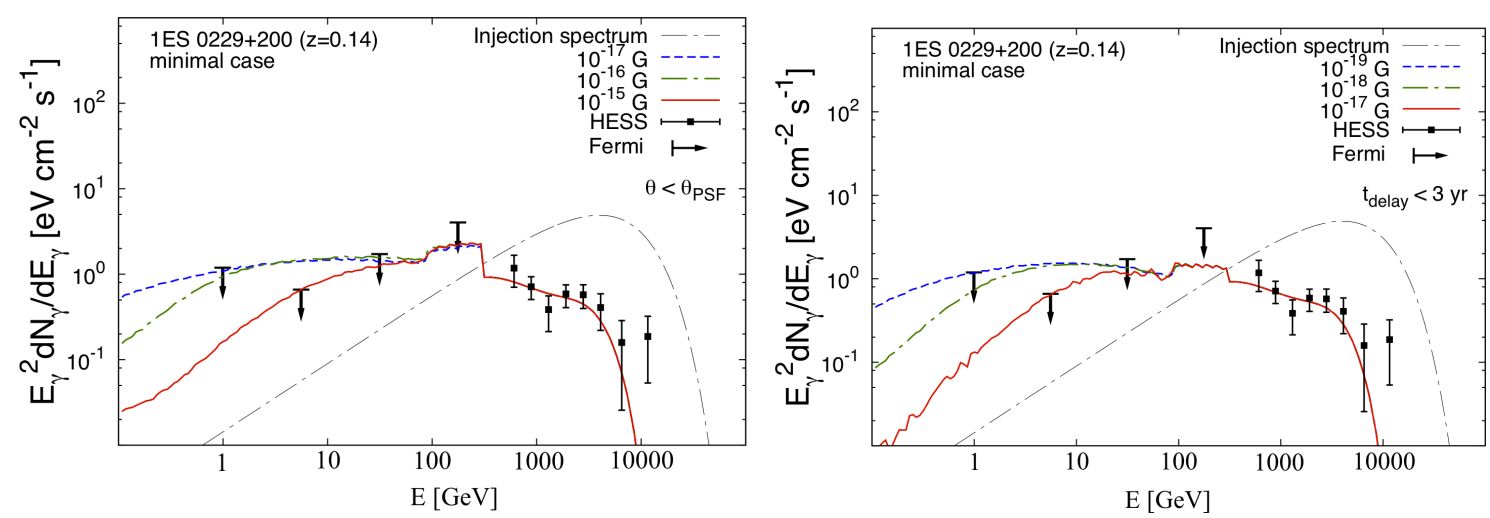

Figure 1: The effects of the presence of an IGMF on the arriving cascade from the blazar 1ES 0229+200. Two panels show the two possibilities of suppression of the cascade flux via extension of the cascade source and time delay of the cascade emission. From [24].

electrons and positrons by the same angle within a given cooling time, see Fig. 2.

The bound $B>10^{-17} \mathrm{G}$ derived from the time delay assumes that the period of activity of a blazar (e.g. 1ES 0229+200) is just of the order of the time span of simultaneous observations in the $\mathrm{GeV}$ and $\mathrm{TeV}$ energy band. It the typical time span of activity of the blazar is much larger than $1 \mathrm{yr}$, the cascade emission could not be suppressed by the time delay effect. Typical estimates of the lifetimes of AGN are $10^{7}-10^{8} \mathrm{yr}$. Assuming that the time of activity of the blazar is much longer than the observation time span, one finds that the only way to suppress the cascade signal is via the extension of the cascade source. This mechanism of suppression was assumed by $[14,18]$ who derived a tighter lower bound $B \geq 3 \times 10^{-16} \mathrm{G}$ for $\lambda_{B} \geq 1 \mathrm{Mpc}$ under this assumption. The suppression of the cascade emission due to the extended nature of the cascade signal in the case of Fermi observations of 1ES 0229+200 is shown in the left panel of Fig. 1. The bound derived under the assumption of suppression of cascade signal due to extended emission also improves as $\lambda_{B}^{1 / 2}$ if the correlation length of IGMF is shorter than $\sim 1 \mathrm{Mpc}$. This bound is shown by the light-blue shading in Fig. 2.

The mean free path of primary multi-TeV $\gamma$-rays is of the order of $\sim 100 \mathrm{Mpc}$. The largest structures in the Universe, galaxy clusters, have typical sizes of the order of several Mpc and their volume filling factor is small. Most of the volume of the sphere of radius $\sim 100 \mathrm{Mpc}$ around TeV blazars is occupied by the voids in the Large Scale Structure. Fig. 2 summarizes the known limits on the strength and correlation length of IGMF in the voids.

The largest possible correlation length of IGMF could be of the order of the Hubble radius. Magnetic fields with such large correlation length could be produced only during Inflation epoch in the Early Universe [25, 26]. Otherwise, magnetic field produced in a causal way at later epochs could not have corrleation length larger than the size of the cosmological horizon at the "magnetogenesis" epoch, typically associated to one of the phase transitions in the Early Universe, such as recombination [27, 28], electroweak [29, 30, 31] and QCD [32, 33, 34] phase transitions. If the cosmological magnetogenesis happens before Recombination, the correlation length of the relic IGMF left from the Early Universe could not exceed the size of horizon at the Recombination. If 


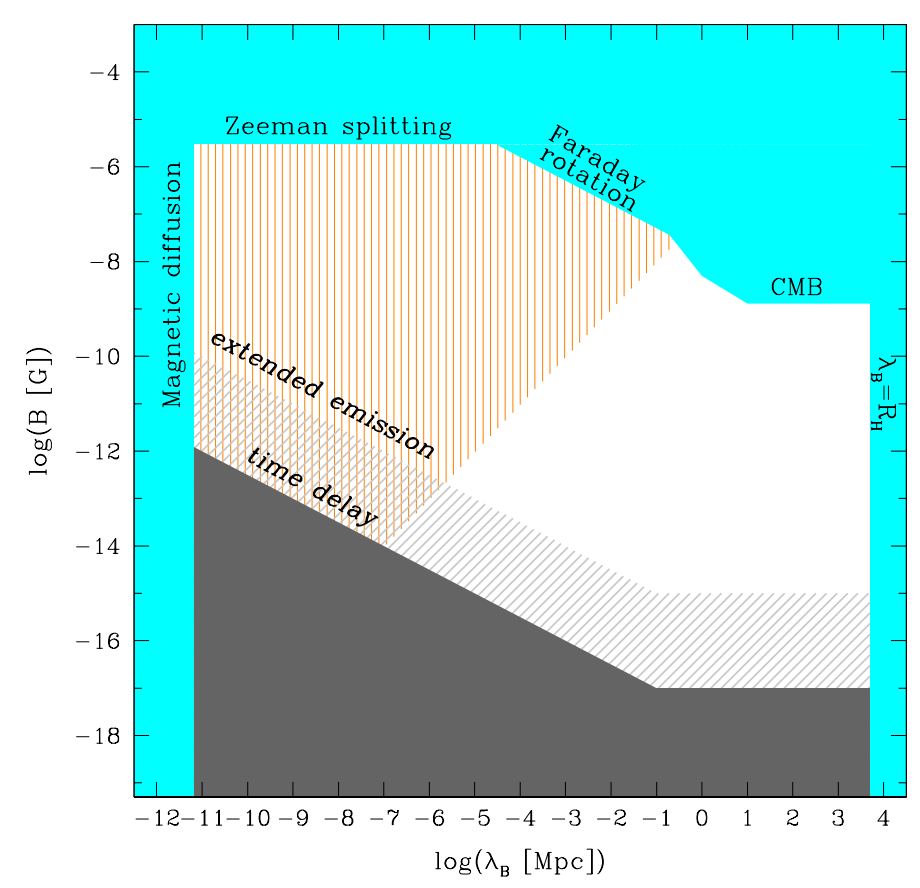

Figure 2: Bounds on magnetic field derived from the simultaneous $\mathrm{GeV}-\mathrm{TeV}$ data. Blue shaded regions show the previously known bounds on $B$ and $\lambda_{B}$, summarized by [14]. Orange shading shows the upper bound on $B, \lambda_{B}$ which could be generated before the epoch of recombination, derived by [41].

IGMF is produced at the stage of structure formation e.g. by the outflows of magnetized plasma induced by supernovae [35], activity of active galactic nuclei [36] or gravitational collapse [6, 7] of proto-galaxies, the correlation length of IGMF should be of the order of typcial correlation length of galactic and clusted magnetic fields, which is in the 0.1-10 kpc range. A lower bound on ISMF correlation length could be derived from the requirement that the resistive dissipaiton time of IGMF is larger than the Hubble time. This gives a lower bound of $\sim 10^{14} \mathrm{~cm}$ [2] shown as a vertical line in Fig. 2.

An upper bound on the IGMF strength could be derived from the natural assumption that the IGMF does not exceed the typical field strength measured in the galaxies (including the Milky Way galaxy) and galaxy clusters. The direct measurements of the field strength in galaxies are possible via the detection of Zeeman splitting of spectral lines [37]. These measurements are sensitive down to the micro-Gauss-scale strength, which could be considered as an absolute upper bound on the IGMF strength. This absolute upper bound does not depend on the correlation length.

An alternative technique used for the measurement of magnetic fields in galaxies and galaxy clusters is via detection of Faraday rotation of the polarization plane of radio emission from distant radio sources (e.g. quasars) [1]. This technique is also sensitive for micro-Gauss-scale magnetic fields if the correlation length is in the range of typical correlation lengths of galactic magnetic fields, $\sim 0.1 \mathrm{kpc}$. Non-observation of IGMF-induced Faraday rotation of polarized emission from distant quasars imposes a limit on IGMF at the level of $\sim \mu G$ for $\lambda_{B} \sim 0.1 \mathrm{pc}$. Contrary to the limit from Zeeman splitting, the Faraday rotation limit depends on $\lambda_{B}$. If $\lambda_{B} \ll R_{H}$, the polarization angle experiences random changes due to the passages of multiple "cells" of the size $R \sim \lambda_{B}$ with 
coherent magnetic field. This means that the the position angle of the polarization plane $\chi$ changes proportionally to the square root of the distance, $\Delta \chi / \Delta \lambda^{2} \sim \sqrt{D \lambda_{B}}$ where $D$ is the distance to the radio source. Non-detection of the EGMF induced Faraday rotation implies, therefore, an upper limits on $B$ which scales as $B \sim \lambda_{B}^{-1 / 2}$. This upper limit is shown in Fig. 2 as an inclined blueshaded region.

If EGMF was produced in the Early Universe before the epoch of CMB decoupling, strong enough IGMF could have influenced the angular power spectrum of CMB fluctuations. In this case a limit on the strength magnetic fields homogeneous over the Hubble distance scale could be derived from the non-observation of the large angular scale anisotropies of the CMB. This gives an upper limit $B \leq 4 \times 10^{-9} \mathrm{G}$ for the fields with $\lambda_{B} \sim R_{H}$ [38]. The powerlaw index dependent limit on the magnetic field strength were derived from the analysis of the CMB angular power spectrum in the Refs. $[39,40]$. The envelope of the upper bounds on $\left(B, \lambda_{B}\right)$ for the range of the powerlaw indices $-3 \leq n \leq 2$ is the lower boundary of the upper bound region of $\left(B, \lambda_{B}\right)$ parameter space marked "CMB" in Fig. 2.

Energy contained in magnetic fields with small enough correlation length is dissipated in the course of evolution of the universe. This leads to the increase of the "integral scale" (i.e. the distance scale which gives dominant contribution to the magnetic magnetic field energy density) with time [41] up to the scale $\lambda_{B, I} \sim v / H$ where $v$ is the characteristic velocity scale, which is of the order of either Alfven or viscous velocity at different epochs of the Universe expansion. Numerically, $\lambda_{B, I} \sim 1\left[B / 5 \times 10^{-12} \mathrm{G}\right] \mathrm{kpc}$ for magnetic fields produced much before recombination epoch and $\lambda_{B, I} \sim 1\left[B / 8 \times 10^{-11} \mathrm{G}\right] \mathrm{kpc}$ for the magnetic fields produced at recombination. This limit on cosmologically produced IGMF is shown as an orange-hatched region in Fig. 2.

Evidence for existence of magnetic fields in the voids provides a new important clue about the origin of the weak seed fields which serve as starting point of amplification in basically all the theoretical models of the origin of magnetic fields in galaxies and galaxy clusters: it is possible that the detected IGMF are just these seed fields in their unamplified form. Further study of IGMF using the gamma-ray observations, possibly in combination with radio and microwave band observations could advance the resolution of the long-standing problem of the origin of cosmic magnetic fields.

\section{References}

[1] Kronberg P.P., 1994, Rept.Prog.Phys., 57, 325

[2] Grasso D., Rubinstein H.R., 2001, Phys.Rept., 348, 163

[3] Widrow L.M., 2002, Rev.Mod.Phys. 74, 775

[4] Beck R., 2009, AIP Conf. Proc. 1085, 83

[5] Kulsrud R.M., Zweibel E.G., 2008, Rept.Prog.Phys., 71, 0046091

[6] Pudritz R.E., Silk J., 1989, Ap.J., 342, 650

[7] Gnedin N.Y., Ferrara A. Zweibel E.G., 2000, Ap.J., 539, 505

[8] Aharonian F.A., Coppi P. Voelk H., 1994, Ap.J., 423, L5

[9] Plaga R., 1995, Nature, 374, 430

[10] Coppi P.S., Aharonian F.A., 1997, Ap.J., 487, L9 
[11] Neronov A., Semikoz D.V., 2007, JETP Lett., 85, 473, [arXiv:astro-ph/0604607]

[12] Murase K., Takahashi K., Inoue S., Ichiki K., Nagataki S., 2008, Ap.J., 686, L67

[13] Eungwanichayapant A., Aharonian F.A., 2009, Int.J. Mod.Phys. D 18, 911

[14] Neronov A., Semikoz D.V., 2009, Phys.Rev. D 80, 123012

[15] Elyiv A., Neronov A., Semikoz D.V., 2009, Phys. Rev. D, 80, 023010

[16] Dolag K., Kachelriess M., Ostapchenko S. and Tomas R., 2009, Ap.J. 703, 1078

[17] Neronov A., Vovk Ie., 2010, Science, 328, 73

[18] Tavecchio F., Ghisellini G., Foschini L., Bonnoli G., Ghirlanda G., Coppi P., 2010, MNRAS, 406, L70

[19] Dermer C.D., Cavadini M., Razzaque S., Finke J.D. Lott B., 2010, arXiv:1011.6660

[20] Gould R.J., Schréder G.P., 1967, Phys. Rev. Lett., 16, 252

[21] Aharonian F.A., et al., 2006, Nature, 440, 1018

[22] Aharonian F.A., et al., A\&A, 475, L9

[23] Franceschini A., Rodighiero G., Vaccari M., 2008, A\&A, 487, 837

[24] Taylor A., Vovk.Ie., Neronov A., 2011 arXiv:2011.???? submitted to A\&A

[25] Turner M.S. \& Widrow L.M., 1988, Phys. Rev. D 37, 2743

[26] Ratra B., 1992, Ap.J. 391, L1

[27] Harrison E., 1970, MNRAS 147, 279

[28] Rebhan A., 1992, Ap.J. 392, 385

[29] Baym G., Bodeker D. \& McLerran L.D., 1996, Phys. Rev. D 53, 662

[30] Vachaspati T., 1991, Phys. Lett. B 265, 258

[31] Grasso D. \& Riotto A., 1998, Phys. Lett. B 418, 258

[32] Quashnock J.M., Loeb A., \& Spergel D.N., 1989, Ap.J. 344, L49

[33] Cheng B.I. \& Olinto A.V., 1994, Phys. Rev. D 50, 2421

[34] Sigl G., Olinto A.V. \& Jedamzik K., 1997, Phys. Rev. D 55, 4582

[35] Rees M., 1987, Q.J. Roy. Astr. Soc., 28, 197

[36] Donnert J., Dolag K., Lesch H. \& Muller E., 2008, arXiv:0808.0919

[37] Heiles C., \& and Troland T.H., 2004, Ap.J.Supp. 151, 271

[38] Barrow J.D., Ferreira P.G. \& Silk J., 1997, Phys. Rev. Lett. 78, 3610

[39] Durrer R., Ferreira P.G. \& Kahniashvili T., 2000, Phys. Rev. D61, 043001

[40] Giovannini M., 2009, Phys. Rev. D 79, 103007

[41] Banerjee R. \& Jedamzik K., 2004, Phys.Rev. D 70, 123003 